\title{
No need for a body model: Positive velocity feedback for the control of an 18-DOF robot walker
}

\author{
Josef Schmitz*, Axel Schneider, Malte Schilling and Holk Cruse \\ Department for Biological Cybernetics, Faculty of Biology, University of Bielefeld, P.O. Box 1001 31, D-33501 Bielefeld, Germany
}

(Received 7 March 2008; final version received 22 May 2008)

\begin{abstract}
In a multilegged walking robot several legs usually have ground contact and thereby form a closed kinematic chain. The control of such a system is generally assumed to require the explicit calculation of the body kinematics. Such a computation requires knowledge concerning all relevant joint angles as well as the segment lengths. Here, we propose a biologically inspired solution that does not need such a body model. This is done by using implicit communication through the body mechanics (embodiment) and a local positive velocity feedback strategy (LPVF) on the single joint level. In this control scheme the locally measured joint velocity of an elastic joint is fed into the same joint during the next time step to maintain the movement. At the same time, an additional part of this joint controller observes the mechanical joint power to confine the positive feedback. This solution does not depend on changes of the geometry, e.g. length of individual segments, and allows for a simple solution of negotiation of curves. The principle is tested in a dynamics simulation on a six-legged walker and, for the first time, also on a real robot.
\end{abstract}

Keywords: positive velocity feedback; hexapod walking; joint coordination; compliant motion; stick insect

\section{Introduction}

Animals and insects show powerful walking capabilities and are capable of dealing with disturbances of different kinds, like uneven terrain, climbing on twigs, losing hold of the ground on slippery surfaces or even losing a leg. Their ability to adapt to changing conditions and to counteract interferences is desired for robots, too. Therefore, it is expected that identifying underlying principles that govern the behaviour of animals can guide the development of walking machines.

Systems controlled by one central controller that regulates all joint movements show a high performance in running on a flat terrain. But when dealing with disturbances these systems need quite a high amount of supervisory control to balance out the disturbance and cannot match the performance of biological systems. Findings on biological systems, i.e. animals like insects, suggest that the strength of these systems does not rely on an accurate calculation of reactions to disturbances but in the modularity of the overall system and by exploitation of the physics of the body. The complexity of the task is distributed onto different levels-it is important to recognise that these levels do not only involve controllers but also mechanical properties, like the elasticity of muscles, which are important in stabilising walking, or interactions with the environment, which can make explicit computations superfluous.

\section{Walknet}

The Walknet is a controller structure which follows the modular approach mentioned above and which is inspired by research on walking stick insects. In fact, it can account for a wide variety of findings with respect to each of the different contributing modules, which we will briefly introduce in the following. In simulations of the walking robot TARRY IIB the Walknet has been tested as being able to control walking behaviour and to deal with disturbances.

The control of walking of a hexapod walker, like the stick insect, involves the control of six legs with three degrees of freedom each (see Figure 1). The Walknet has been introduced to control such an insect-like artificial structure consisting of six legs with three hinge joints. The configuration of this robot mimics the stick insect with respect to its dimensions, position and orientation of the joints. This results in a robot with a total of 18 degrees of freedom that have to be controlled in parallel. It seems natural to divide this control problem up into smaller parts. Biological findings (Cruse 1990; Bässler and Büschges 1998; Dürr et al. 2004) suggest that each leg has its own controller structure, which only has to deal with three joints (Figure 2). This simplifies the control dramatically: On the level of the leg we have only two movements to distinguish - the stance and the swing movements - and we need some kind of switch that decides which of both controllers should be active at the moment. The movement controllers themselves

*Corresponding author. Email: josef.schmitz@uni-bielefeld.de 


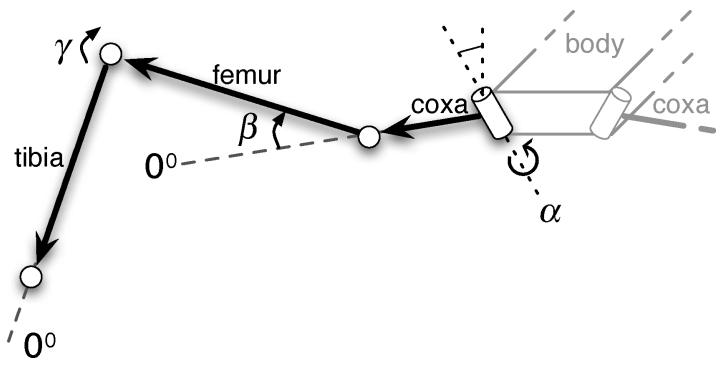

Figure 1. Schematic figure of an insect leg (right front leg). It consists of three hinge joints. The $\alpha$-joint moves the leg for- and backward (protraction means positive direction). The axis of this joint is slanted. Its articulation with respect to the body coordinate system can be described by two angles $\varphi$ and $\psi$ (for further detail see text). $\beta$ - and $\gamma$-joints operate in the leg plane, meaning their axes are parallel to each other and are perpendicular to the drawn leg plane. Lifting the leg equals a positive movement in the $\beta$-joint, an outward going movement produced by the $\gamma$-joint (extension) is defined as a positive movement. The origins of the joint coordinate systems are set corresponding to leg positions in a standing walker ( $\alpha$ is in a middle position, while $\beta$ - and $\gamma$-joints are in a position in which the femur is approximately parallel to the ground and the tibia is nearly orthogonal to the femur.

are modelled as neural networks. To be able to react to external disturbances these neural networks cannot be driven in an open-loop way, but need some form of feedback. For the swing movement a feedback loop through the environment is sufficient. The stance movement seems more difficult because all the joints of all standing legs are interacting and influencing each other. The latter problem concerns the main question dealt with in this paper. In Section 3 the local positive velocity feedback will be introduced, which controls the joints on a local level using recurrent connections and the feedback through the environment.

A switch - called selector - alternately activates the swing and stance behaviours leading to a rhythmical movement of one leg. The decision which behaviour to choose is based on the one hand on the current state of the leg and on the other hand depends on the state of the other legs. Different coordination rules acting only between neighbouring legs have been found in experiments on stick insects and are applied in the Walknet to coordinate the behaviour of all the legs.

\subsection{Selector}

Each leg has its own pattern generator, which is continuously switching between the two possible leg states - swing and stance. In the Walknet controller described here an analog selector is applied (see Figure 3, a detailed explanation can be found in Schilling et al. [2007]) which not only selects which behaviour has to be activated but also how strong it should be activated.

The decision process depends on different variables.

- On the one hand there is the current state of the leg. Feedback connections within the selector enforce the network to remain in the current state and suppress the other state leading to a stable behaviour.

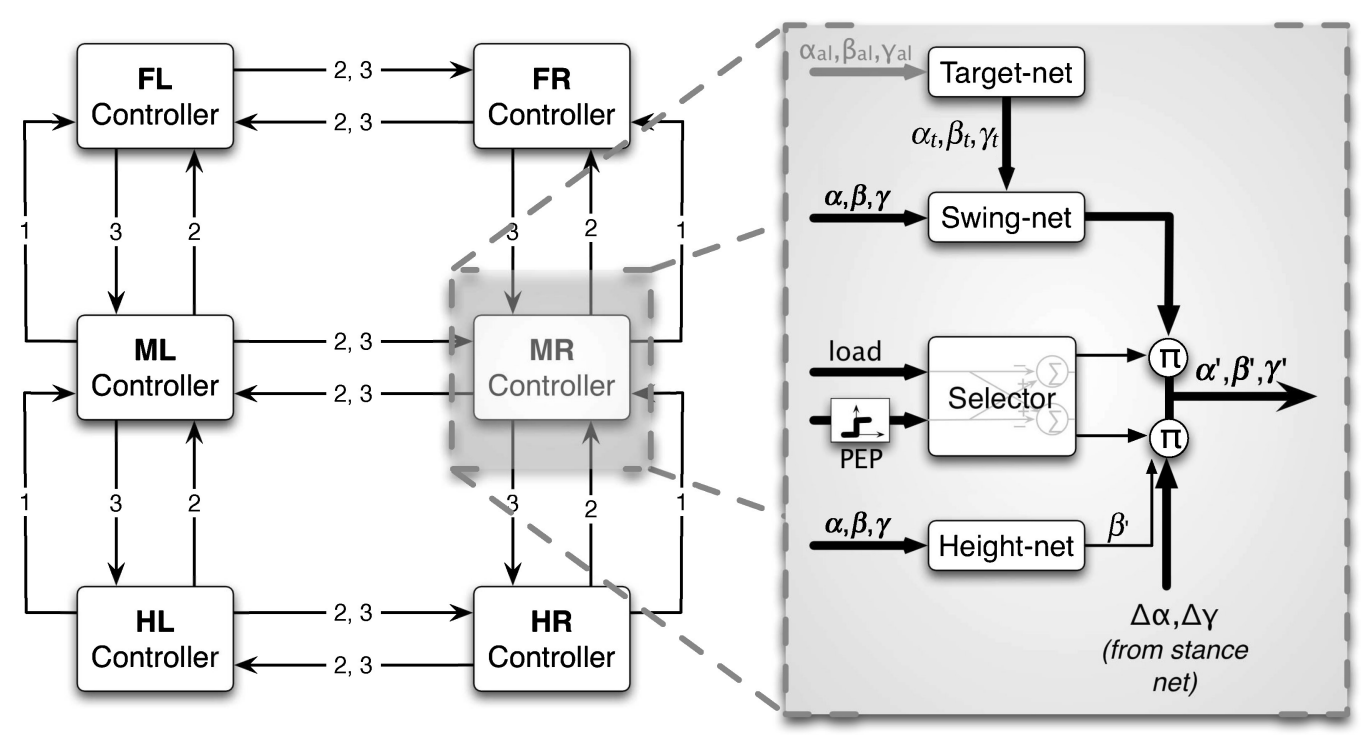

Figure 2. General architecture of the Walknet that is able to control six-legged walking. The system consists of six independent controllers (left part), one for each leg (FL/FR left/right front leg, ML/MR left/right middle leg, HL/HR left/right hind leg). Each leg controller contains several modules (right part): a swing- and a stance-net to control swing and stance movements, respectively. A height-net for regulating body ground distance during stance, a target-net for determining the anterior extreme position of the swing movement and a selector-net that decides at what time the swing-net or stance-net has access to the motor output $\left(\alpha^{\prime}, \beta^{\prime}, \gamma^{\prime}\right) . \alpha, \beta$ and $\gamma$ denote the three joint angles of a leg. Influences $(1,2,3)$ that change the posterior extreme position (PEP) of the leg (shown on the left side of the figure) act only between neighbouring legs. 


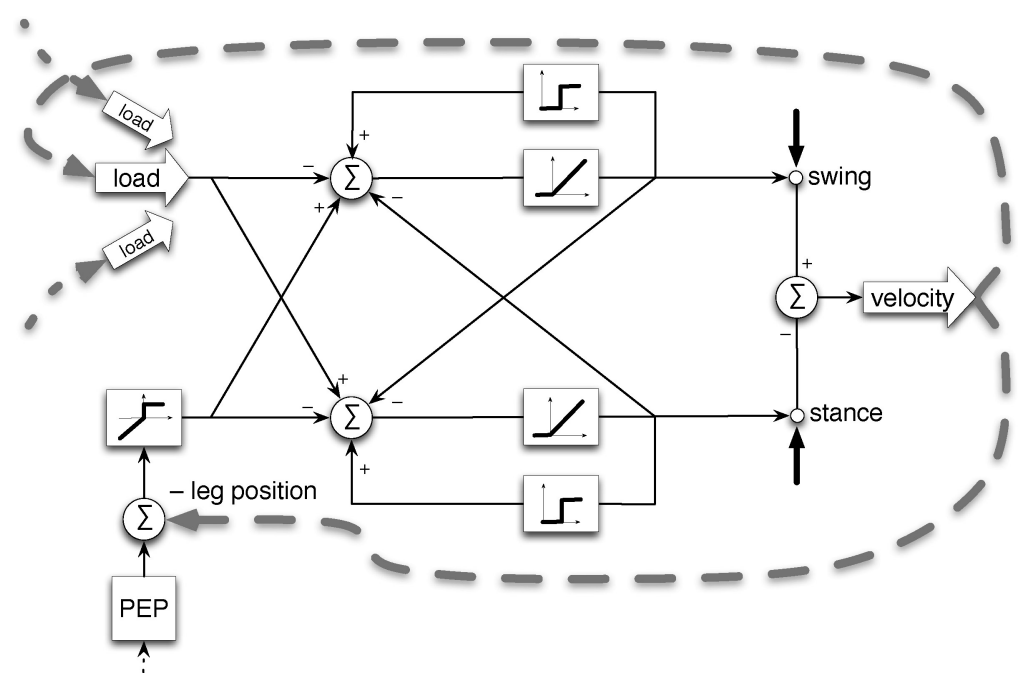

Figure 3. The wiring of the analog selector: upper left: load input from the own and other legs; lower left: position input and PEP that can be influenced through the coordination influences. The two outputs (right) represent analogous control signals for the stance or swing movements, stance and swing, respectively. Bold arrows on the right indicate connections from the swing and stance-net, respectively (see Figure 4).

- On the other hand there are sensory inputs, like the position of the leg, ground contact or load on the leg, all of which can also be found in the insect. These sensory inputs guide the overall behaviour of the selector module and particularly invoke a switching between the two states. The swing movement is terminated and the stance movement is started when the leg hits the ground. This is signalled by ground contact sensors and/or load sensors. The transition from stance to swing is initiated when the leg has reached its posterior extreme position (PEP), therefore, depending on the position of the leg.

\subsection{Coordination}

The PEP may be assumed as being fixed in normal walking, but biological investigations on the stick insect have shown that the step length can be varied and that the PEP depends on the state and position of the neighbouring legs. The phases of the legs can be adjusted in a simple way. Six different coordinating influences have been observed in the stick insect and applied to the model (Kindermann 2002; Dürr et al. 2004).

(1) From rear to front: if a sender leg is swinging, the leg in front should not start to swing. This avoids instability.

(2) From rear to front: on touch down of the posterior leg, the anterior leg is stimulated to start swinging. This favours temporal coherence.

(3) From front to rear: while the anterior leg is approaching its PEP, it is stimulating its posterior neighbour to swing, so when the anterior leg starts to swing, the posterior leg may have already finished its swing movement. This is maintaining temporal coherence.

(4) Aiming behaviour: the anterior extreme position (AEP) of a leg is determined by the current position of the anterior leg.

(5) Propulsive force is distributed on neighbouring legs.

(6) Another rule, the treading-on-tarsus reflex will not be considered here (see Schmitz and Haßfeld 1989).

Rules two, three and five are assumed also to operate between contralateral legs (Figure 2). Earlier simulations have shown that these rather simple rules are sufficient to generate a stable walking behaviour: after randomising the positions of the legs, a simulated animal can nearly immediately (in one or two steps) return to a stable gait.

\subsection{Swing movement}

As mentioned, a step consists of two distinct phases: the stance movement and the swing movement. After the leg has reached its PEP, it is lifted from the ground and, during swing, is moved to its AEP. In stick insects this position varies depending on the position of its anterior neighbour. The posterior leg aims at the current position of the anterior leg. This is especially reasonable in climbing in twigs because it guarantees that the leg will find a foothold. The aiming behaviour is described by the coordination rule number 4 .

The swing movement can be controlled by a simple feedforward neural network. Different versions have been applied in the Walknet. The simplest one consists of three neurons for the swing network itself and three additional 
units for a target network. In some more refined versions additional units are introduced which allow avoidance reflexes, searching movements and steadier movements, but even these versions consist in a total of not more than 12 units and 12 connecting weights. In Schumm and Cruse (2006) a version is introduced that uses antagonistic structures allowing for insect-like adaptations of swing movements to varying shapes of the substrate. Recurrent connections and an internal feedback are not needed and can be completely replaced through the sensory inputs from the leg itself that close a feedback loop through the environment (Dürr et al. 2004).

\subsection{Stance movement}

The configuration of the robot depends on the number of standing legs. In normal walking usually three or four legs are touching the ground at the same time, in this way forming a closed kinematic chain. They are coupled through the ground and the body, and can thereby influence each other. To make things more complicated, the configuration and the set of standing legs continuously change over time. At first it seems hard to realise how a controller can deal with this problem only on a local level regarding only one leg and its joints. But while for a central controller (which has to take between nine and 18 joints into account simultaneously) the inclusion of properties of the body and actuators, like elasticity of muscles, makes the calculation of valid control signals very hard or even intractable, these body properties at the same time introduce a form of soft constraints. These constraints are introducing a form of fault tolerance (an example are muscles which can compensate small disturbances through their passive properties). As a consequence, the control of the stance movement is not forced to provide mathematically exact solutions, but approximate signals are sufficient. For previous Walknet versions we have shown that the stance movement can be controlled by simply pushing the leg backwards through the $\alpha$-joint, and regulating the body height through the $\beta$-joint (Cruse et al. 1995; Kindermann 2002; Dürr et al. 2004).

Although such a simple controller approach was able to lead to stable gaits, it does not take the movements of the other legs into account. Following the embodiment approach, we therefore will adopt the local positive velocity feedback (LPVF) approach for the control of the stance movement. This means, that the influences between standing legs are not computed in an explicit way, but are mediated through the body. In the following section the LPVF will be introduced in general and then applied as a controller for the stance movement.

It is this part that is essentially changed compared to earlier versions of Walknet in which simple local controllers have been used to propel the body by a movement of the $\alpha$-joint and maintained stability and body height through simple feedback controllers.

\section{Local positive velocity feedback}

To maintain their posture during standing, all animals investigated show resistance reflexes in their joints. These reflexes counteract external forces disturbing the stability of the animal. Resistance reflexes involve negative feedback control loops acting on the angular position of a given joint.

Physiological studies on the stick insect Carausius morosus have revealed that, for the $\gamma$-joint, during the stance phase of walking animals, the resistance reflex is reversed into an assistance reflex (Bässler 1976). In this case, those muscles are activated that assist the externally induced joint movement. Reflex reversal is assumed to occur if the external force acting on the leg results in a joint angle acceleration below a certain threshold (Bartling and Schmitz 2000). It has been suggested that positive feedback on the single joint level is responsible for the reflex reversal effect (Bässler 1986). Such reflex reversals have been observed in the $\alpha$ - and the $\gamma$-joints, but not in the $\beta$-joint (Schmitz et al. 1995). Kinematic simulations have shown that positive feedback in the body - coxa $(\alpha-)$ and femur - tibia $(\gamma-)$ joints is sufficient to produce realistic stance movements in a virtual walking agent (Schmitz et al. 1995). However, to generate considerable mechanical power in a real multibody system afflicted with friction and a considerable mass, only a specific implementation of local positive feedback, namely power-controlled local positive velocity feedback (LPVF), is able to generate meaningful joint movements (Schneider et al. 2006). In the present study, power-controlled LPVF is used to move the $\alpha$ - and $\gamma$-joints of all legs with ground contact in a dynamics simulation of the six-legged robot TARRY IIB $(\beta$-joints are controlled through negative feedback by the height-net to keep the body clearance constant).

The general function principle of LPVF can be formulated as follows. During the stance movement, all legs that touch the ground form closed kinematic chains. This means that no joint can be moved without influencing all other joints in the chain. If the body of the walker is moving forward (mechanical impulse) and a leg touches the ground (and thus becomes member of a closed kinematic chain), the elastic actuators of the $\alpha$ - and $\gamma$-joints experience some bending, which is built up by the passive joint movements. In the described situation, the LPVF controlled joint has to comply with the following three requirements to generate a powerful stance movement without mechanically overloading other joints in the chain.

(1) The joint has to be elastic to accept the externally induced bending (passive compliance). 


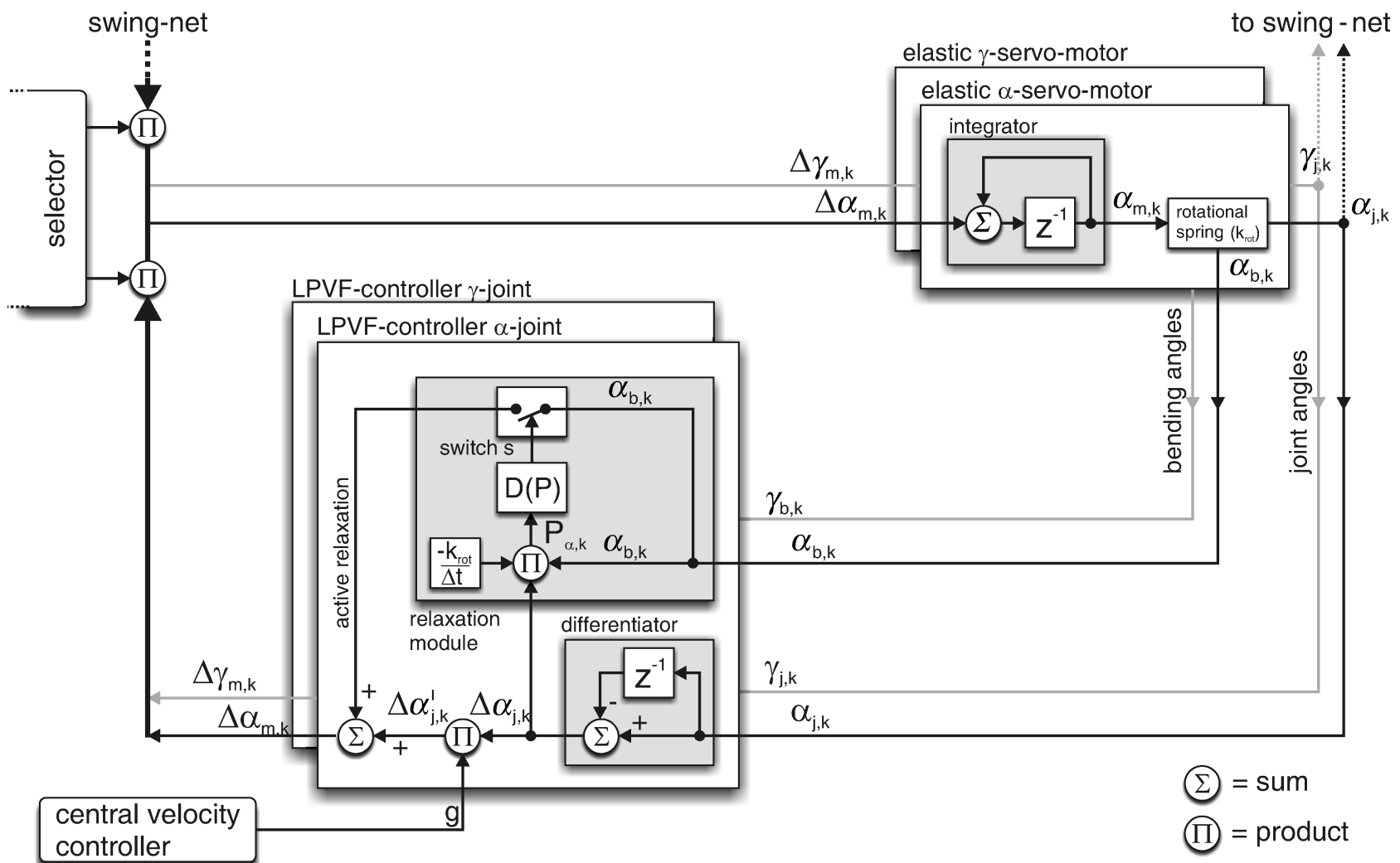

Figure 4. Schematic drawing of the stance-net consisting of two LPVF control-circuits for the $\alpha$ - and $\gamma$-joints, respectively. $z$ is the time-shift operator.

(2) The LPVF joint controller has to actively relax some part of the joint bending to avoid mechanical overload (active compliance, active relaxation). At the same time, a rest of bending (torque) has to be maintained to transmit mechanical power to the leg and to propel the body forward.

(3) The passive joint movement already indicates the direction and amplitude of an active joint movement in the next time step. This active joint movement is generated by the LPVF controller via feeding the passive movement back into the joint actuator (positive velocity feedback).

Figure 4 shows the implementation of the above idea for the $\alpha$ - and $\gamma$-joints of a single leg. The following explanation will be given for the $\alpha$-joint as an example, but is also valid for the $\gamma$-joint. Discrete time steps are indicated by the index $k$. The time-shift operator is represented as $z^{-1}$. Indices $j, m$ and $b$ stand for joint, motor and bending, respectively.

We begin with the elastic servo-motor in the upper right corner of Figure 4. The actual servo-motor is represented by an integrator circuit that accumulates the velocity commands $\Delta \alpha_{\mathrm{m}, \mathrm{k}}$ at the motor input to generate a new axis angle $\alpha_{\mathrm{m}, \mathrm{k}}$ at the motor output. A rotational spring with a spring constant $k_{\text {rot }}$ is connected in series to the motor axis and acts on the next segment. If an external torque $\tau$ affects the joint the rotational spring is twisted by a rotational bending angle $\alpha_{\mathrm{b}, \mathrm{k}}$. Figure 5 shows the implementation of this idea in the robot TARRY IIB for the $\alpha$ - (a) and $\gamma$-joint (b), respectively. In the $\alpha$-joint, the rotational spring is realised with a spring-steel wire, and in the $\gamma$-joint with two extension springs.

The absolute joint angle of the output axis is determined by

$$
\alpha_{\mathrm{j}, \mathrm{k}}=\alpha_{\mathrm{m}, \mathrm{k}}+\alpha_{\mathrm{b}, \mathrm{k}}
$$

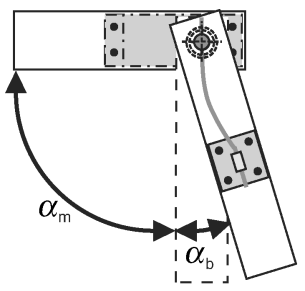

(a)

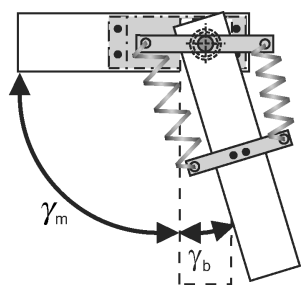

(b)
Figure 5. Schematic drawings of the elastic servo-motors (compliant joints) as implemented in TARRY IIB for the $\alpha$ - (a) and $\gamma$-joints (b), respectively. 


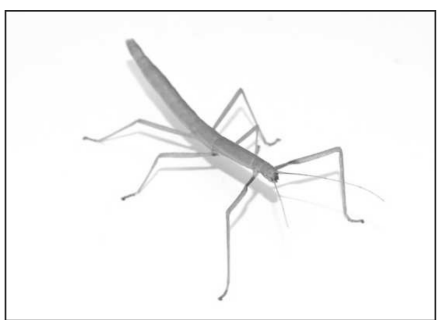

(a)

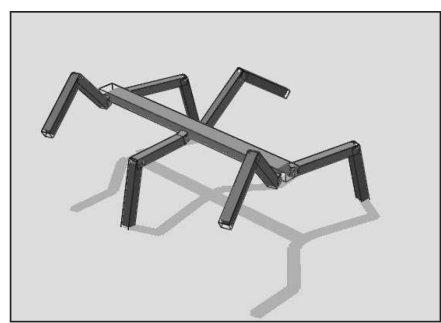

(b)

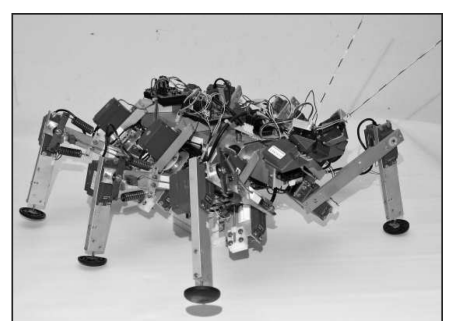

(c)

Figure 6. (a) Model system, the stick insect Carausius morosus. (b) Simulation model in Breve (1:10 scale) according to the size and weight of the robot TARRY IIB, which is shown in (c). Photographs courtesy of Wolfram Schenck.

The joint angle $\alpha_{\mathrm{j}, \mathrm{k}}$ and the bending angle $\alpha_{\mathrm{b}, \mathrm{k}}$ are fed into the LPVF - controller of the $\alpha$-joint in the lower left corner in Figure 4. The joint angle $\alpha_{j, k}$ is connected to the input of the differentiator. The output of the differentiator delivers the current joint velocity $\Delta \alpha_{\mathrm{j}, \mathrm{k}}$. This joint velocity can be altered by a signal of a central velocity controller that controls the forward velocity $v_{\text {body,k }}$ of the walker by changing the gain $g$. The desired body velocity is $v_{\text {body,des }}$. The output of the central velocity controller delivers the following output signals that are always close to 1 :

$$
g=\left\{\begin{array}{lll}
>1 & : & \text { if } v_{\text {body,des. }}>v_{\text {body,k }} \\
=1 & : & \text { if } v_{\text {body,des. }}=v_{\text {body,k }} . \\
<1 & : \quad \text { if } v_{\text {body,des. }}<v_{\text {body, } \mathrm{k}}
\end{array}\right.
$$

The altered joint velocity can be written as

$$
\Delta \alpha_{\mathrm{j}, \mathrm{k}}^{\prime}=g \Delta \alpha_{\mathrm{j}, \mathrm{k}}
$$

To produce the next motor command $\Delta \alpha_{\mathrm{m}, \mathrm{k}}$ for the servo motor input we add the active relaxation signal to the altered joint velocity $\Delta \alpha_{\mathrm{j}, \mathrm{k}}^{\prime}$. This active relaxation signal is produced in the relaxation module. It consists of the current bending angle $\alpha_{\mathrm{b}, \mathrm{k}}$ of the elastic servo-motor (twist of the rotational servo spring). The relaxation signal is turned on and off by a switch $s$. This switch is operated by a decision function $D(P)$ in dependence of the current mechanical power that is generated by the joint.

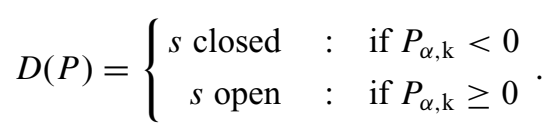

The mechanical power of the rotational joint can be calculated as

$$
P_{\alpha, k}=\frac{\Delta \alpha_{\mathrm{j}, \mathrm{k}}}{\Delta \mathrm{t}} \cdot \tau_{\alpha, \mathrm{k}}=-\frac{\Delta \alpha_{\mathrm{j}, \mathrm{k}}}{\Delta \mathrm{t}} \cdot k_{\mathrm{rot}} \alpha_{\mathrm{b}, \mathrm{k}} .
$$

with $\tau_{\alpha, \mathrm{k}}$ being the joint torque, $\Delta t$ the sample time of the system and $k_{\text {rot }}$ the spring constant of the actuator. A positive mechanical joint power means that the actual angular velocity originates from the torque exerted by the elastic servo-motor of the joint. However, a negative mechanical power represents the fact that the directions of joint torque and joint rotation are opposed to each other. In the negative power case, the joint has to behave compliantly (active relaxation) until it generates positive mechanical power again. The decision function in Equation (4) operates the switch $s$ according to the behaviour as described above.

\section{Dynamics simulation and implementation on a robot}

The principle of reflex reversal (assistance reflex) has been found in the movement system of different species. In the standing locust it is involved in the preparation of a kicking movement (Burrows and Pflüger 1988), Vedel (1980) found reflex reversals in the antennal motor system of the rock lobster, DiCaprio and Clarac (1981) demonstrated that a passive movement of the basal (thoracic - coxal) leg joint of the shore crab Carcinus maenas leads to an assistance reflex depending on the activation state of the animal. The discovery of reflex reversal in the femur-tibia $(=\gamma-)$ joint (Bässler 1976, 1988; Bässler and Büschges 1990; Schmitz et al. $1995)$ and the thorax $-\operatorname{coxa}(=\alpha-)$ joint (Schmitz et al. 1995) of the stick insect Carausius morosus (Figure 6a), led to the formulation of the hypothesis that positive (velocity) feedback in these joints underlies the stance movement generation of the corresponding leg (Cruse et al. 1995). The important aspect of this idea is the interaction of all legs on ground via a real(istic) physical environment. LPVF as a sustainable technical implementation of reflex reversal for the movement generation has already been proven to be successful in dynamics simulations of a one-legged preparation and a cranking arm setup (Schneider et al. 2006). In the present study, for the first time, this hypothesis is tested on a dynamics simulation of a six-legged walker (Figure $6 \mathrm{~b}$ ) and on the real six-legged robot TARRY IIB (Figure 6c).

The dynamics simulations were implemented and conducted in BREVE (version 2.6 (Klein 2003)). BREVE is an 

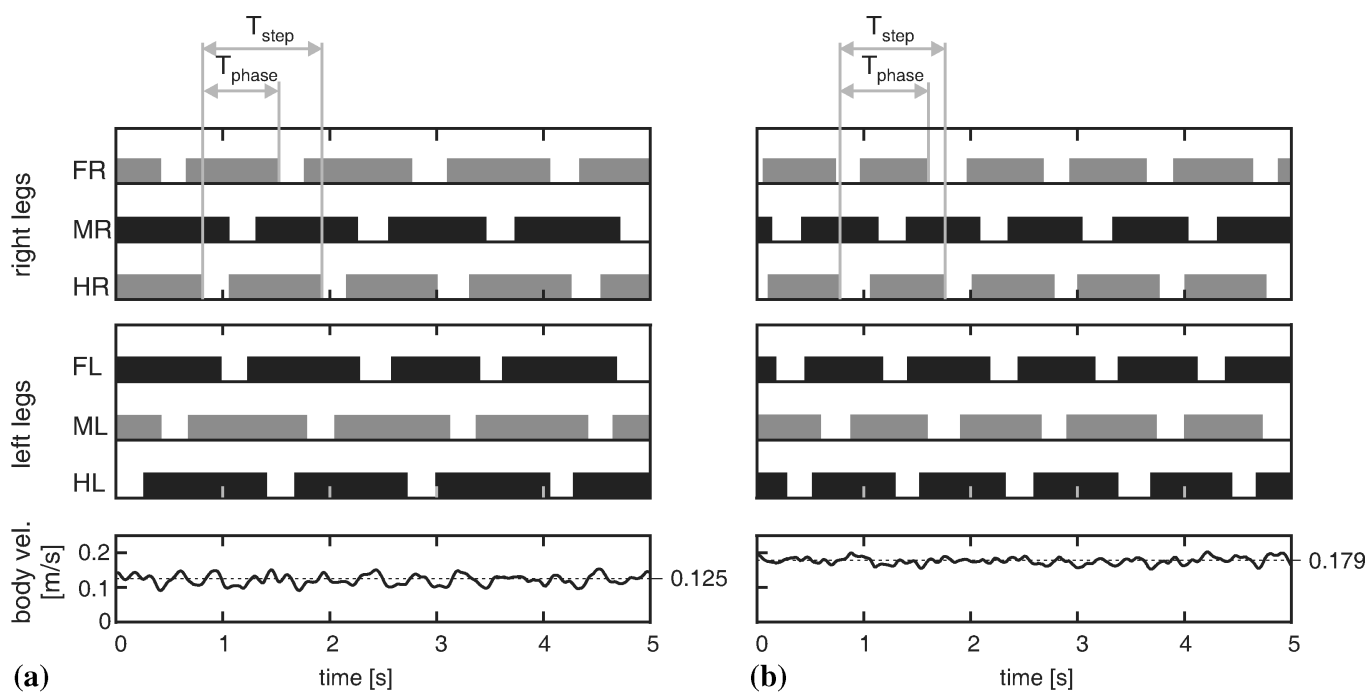

Figure 7. (a) Tetrapod step pattern from the dynamics simulation of a six-legged walker. The desired forward velocity of the central body was $0.13 \mathrm{~m} / \mathrm{s}$ (bottom). (b) Approximate tripod step pattern from the same simulation as a result of an increased desired body velocity of $0.18 \mathrm{~m} / \mathrm{s}$ (bottom).

integrated environment using OpenGL for visualisation and the ODE library for the simulation of the solid state dynamics of the body, legs and the environment. The walker is a scaled-up version of a stick insect (10 times) and matches roughly the dimension and weight of the robot TARRY IIB on which the LPVF approach was also implemented (Section 4.2). For the dynamics simulation the weight of the central body was set to $1 \mathrm{~kg}$ and the weights of the segments (coxa, femur, tibia) were set to $0.1 \mathrm{~kg}$, each. The slanted axes of the $\alpha$-joints (see Figure 1) show leg specific values. In the front legs the axes are tilted outwards by $\psi=45^{\circ}$ and then rotated forward by $\varphi=+5^{\circ}$. The respective angles for the middle legs are $\psi=45^{\circ}$ and $\varphi=-10^{\circ}$, and for the hind legs $\psi=45^{\circ}$ and $\varphi=-25^{\circ}$.

\subsection{Six-legged walking with LPVF in a dynamics simulation}

Figure 7 shows walking sequences corresponding to a typical tetrapod gait in (a) and a tripod gait in (b). Stance phases are indicated by bars. These sequences were taken from the middle section of longer test runs of the dynamics simulation.

As can be seen, proper leg coordination is maintained throughout the walk for both gaits. The desired forward velocity of the body was controlled by the central velocity controller as introduced in Figure 4 (bottom). The desired velocity was first set to $0.13 \mathrm{~m} / \mathrm{s}$, which resulted in the tetrapod gait in Figure 7a. The lower panel of Figure 7a shows that the mean value of the actually achieved body velocity was $0.125 \mathrm{~m} / \mathrm{s}$. An increase of the desired body velocity to $0.18 \mathrm{~m} / \mathrm{s}$ shifted the gait pattern from tetrapod to tripod (Figure 7b). The mean value of the actually achieved body velocity was $0.179 \mathrm{~m} / \mathrm{s}$.

To classify the two gaits, the mean relative phase shift $p$ was calculated for both situations. We define this phase shift as the quotient of the time difference between liftoff of a hind leg and the liftoff of the corresponding ipsilateral front leg $T_{\text {phase }}$ and the total duration of one-step cycle of the hind leg $T_{\text {step }}$ (both values are indicated in Figure 7). For the tetrapod gait, $p$ was 0.66 and for the tripod gait, $p$ was 0.81 (the ideal value for tripod would be 1).

Figure 8 shows the leg trajectories during stance in top view. In agreement with earlier insect studies (e.g. [Cruse 1976; Kindermann 2002]) front leg trajectories are comparatively less regular, middle leg trajectories are about parallel to the body long axis, whereas hind legs usually show a trajectory that approximates a segment of a circle centered at the leg basis. A conspicuous feature of all trajectories concerns their generally irregular shape, which is different to that shown by traditional walking machines, but is quite similar to trajectories observed in insects. All insects show slight side-to-side oscillations of the central body during each step cycle.

More details are shown in Figures 9-11, where time courses of $\alpha$ - and $\gamma$-joint angles and their joint torques are presented for the front, middle and hind leg, respectively.

The $\beta$-joint is not shown as it is due to a traditional negative feedback controller as mentioned above. The $\alpha$ joints, in all three leg types, show the expected behaviour consisting of a strong protraction at the beginning of swing phase that decelerates near its end and then retracts during stance. During stance in front legs there is considerable retraction torque (negative values) that propels the body 


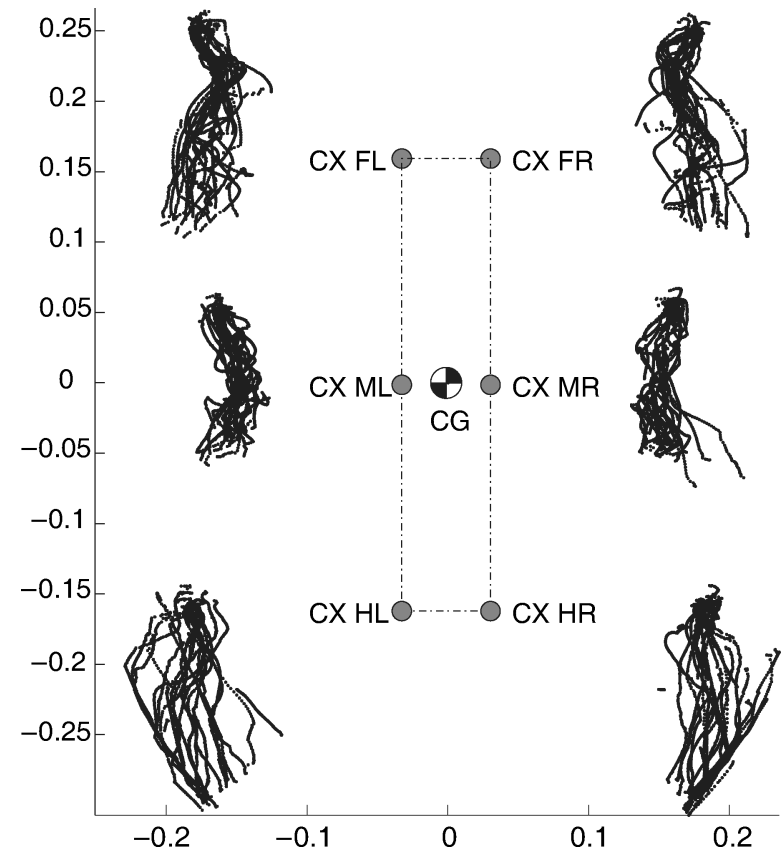

Figure 8. Coordinates of all six tarsus points during the stance phases of one simulation run. The center of gravity is labelled with CG. All coxae (CX) are denoted with their attachment points at the body (FL/FR left/right front leg, ML/MR left/right middle leg, HL/HR left/right hind leg).

forward, in the middle leg the torque is weaker but the mean value is also suited to propel the body. In hind legs the retraction torque is weak for the first few steps and stronger in the last steps. However, on average there is a clear retraction torque, in particular, in the second half of stance movement.

The time courses of the $\gamma$-joints are much more irregular, but again correspond to the behaviour of femur-tibia joints observed in stick insects. During stance the $\gamma$-joint movement in the front and middle legs consists of a flexion followed by an extension in agreement with mean values of experimental results ([Cruse and Bartling 1995], Figure $5 b)$. However, in individual steps there may be more than one of these reversals. In the hind leg, the angle of the $\gamma$ joint appears to be held quite constant in the first quarter of stance. During the remaining time interval the $\gamma$-joint shows an extension. This agrees with the behaviour found in stick insect.

The torques of the $\gamma$-joints vary a lot due to the sidewards movement of the walker during the interaction of all legs in stance. In the hind leg, there is a short flexion torque at the beginning of each stance to support the weight of the body. This supporting phase is longer in the animals. As opposed to stick insects that have their center of gravity (CG) between the coxae of the hind legs, the walker in the dynamics simulation has its CG between the coxae of the middle legs. The extension torque in the $\gamma$-joint during the rest of stance propels the body forward.
In middle legs $\gamma$-torques show a tendency to flexion. This agrees with experimental findings from stick insects. In contrast to the stick insect results, which show flexor torques in front as well as in hind legs, the simulation produces mainly extensor torques in both legs. Whereas in front legs this effect is not obvious for each step due to considerable variation, hind legs always show extensor torques.

\subsection{Six-legged walking with $L P V F$ on a real robot}

To test the feasibility of the power-controlled LPVF approach on a real physical artifact, we implemented this stance controller on the walking robot TARRY IIB. In contrast to the idealized assumptions of the dynamics simulation, the weight distribution of the real robot is not uniform. Due to electronic boards and cables the center of gravity (CG) is shifted posteriorly towards the hind legs. Also the maximum speed of the real motors are such that when approximating a fast walking gait (i.e. ratio swing:stance near 1) the maximal stepping frequency is limited to $2 \mathrm{sec}$ per step. The gait generated was a tripod with the ipsilateral front and hind legs moving in phase and in antiphase to the ipsilateral middle leg.

Figures 12-14 show the time courses of the $\alpha$ - and $\gamma$ joint angles and their torques for several steps of the front, middle and hind legs of the walking robot. $\alpha$ - and $\gamma$-joints were controlled by LPVF during stance while each $\beta$-joint was subject to classical, negative feedback height control (data not shown).

The $\alpha$-joints show a fast protraction movement during swing that decelerates near the swing - stance transition before the LPVF - controller comes into play. During stance, all $\alpha$-joints produce backward movements of their respective leg. According to the situation of the stick insect, the working range of the hind leg is mainly posterior to the hind leg coxa while front and middle legs are moved more or less symmetrically around the $0^{\circ}$ position. Please keep in mind that due to the slanted axes of the body-coxa joints (see Figure 1) the foot of the front leg operates clearly ahead of the front leg coxa while the foot of the hind leg is behind the hind leg coxa. The middle leg operates nearly symmetrically around a position that is perpendicular to the longitudinal body axis.

During stance the $\alpha$-joint movements in front and middle legs produce considerable retraction torques suited to propel the body forward. Hind legs, in contrast, produce retraction torques at the very beginning of each stance only. During the remainder strong protraction torques are produced. This is plausible because the hind legs, due to the posterior working range and the weight distribution along the central body, have to carry a considerable amount of the body weight.

The $\gamma$-joint movements are quite similar to those of insects. During stance, front legs are flexing throughout 


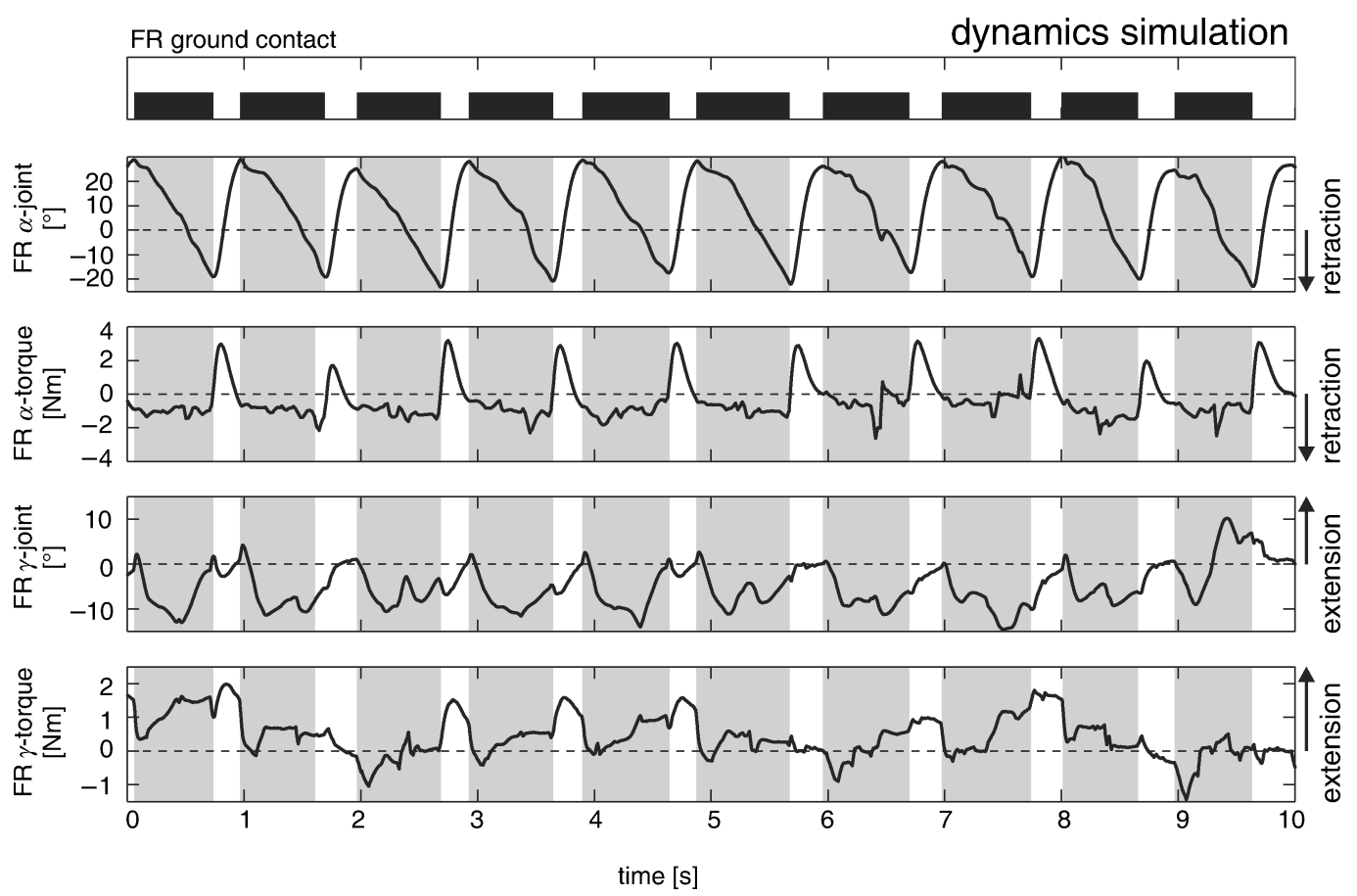

Figure 9. Step pattern of the right front leg in the dynamics simulation (top). Below are given the courses of the $\alpha$-joint angle, the torque of the $\alpha$-servo-drive, the $\gamma$-joint angle and the torque of the $\gamma$-servo-drive (from top to bottom).

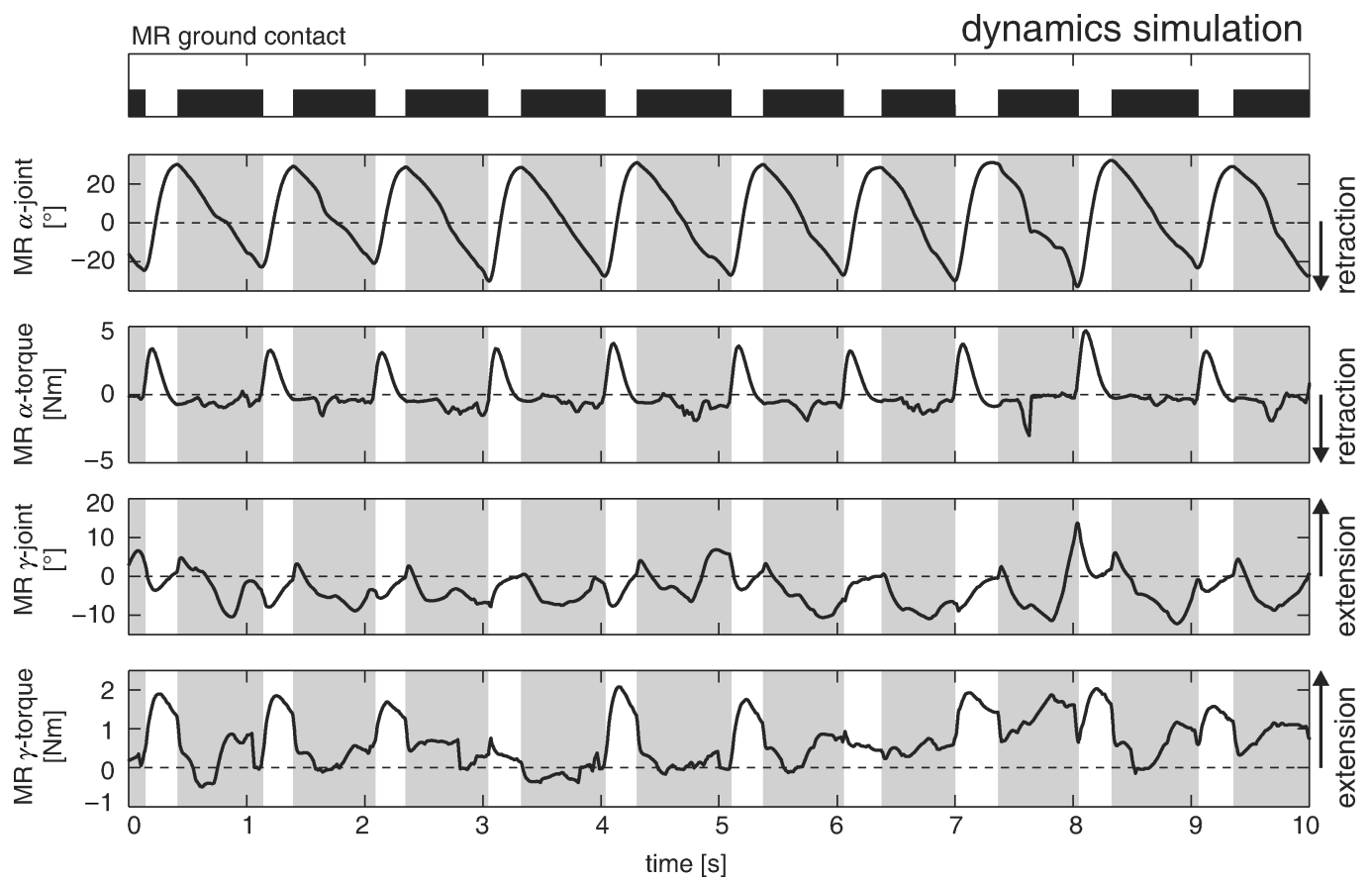

Figure 10. Step pattern of the right middle leg in the dynamics simulation (top). Below are given the courses of the $\alpha$-joint angle, the torque of the $\alpha$-servo-drive, the $\gamma$-joint angle and the torque of the $\gamma$-servo-drive (from top to bottom). 


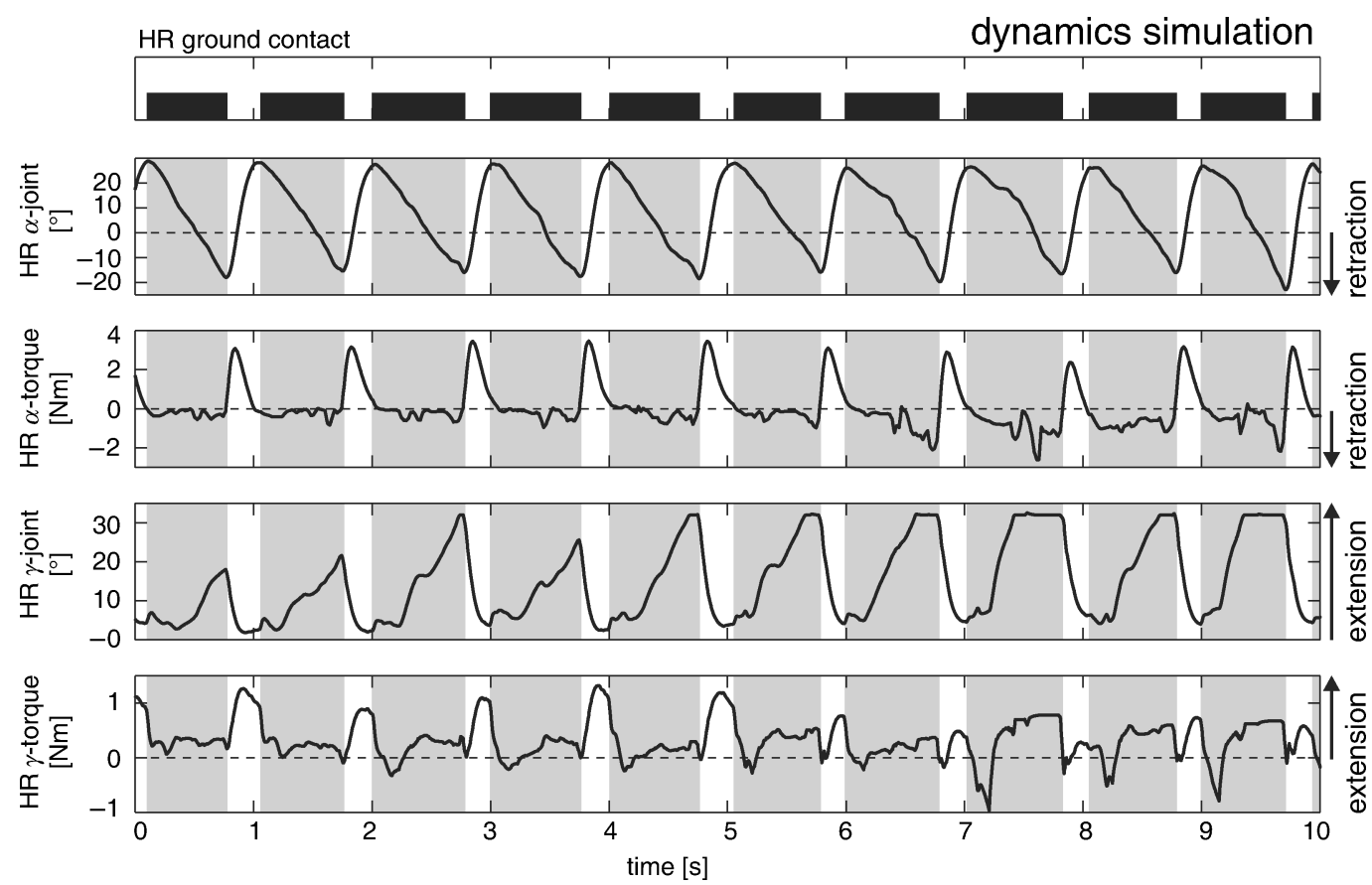

Figure 11. Step pattern of the right hind leg in the dynamics simulation (top). Below are given the courses of the $\alpha$-joint angle, the torque of the $\alpha$-servo-drive, the $\gamma$-joint angle and the torque of the $\gamma$-servo-drive (from top to bottom).

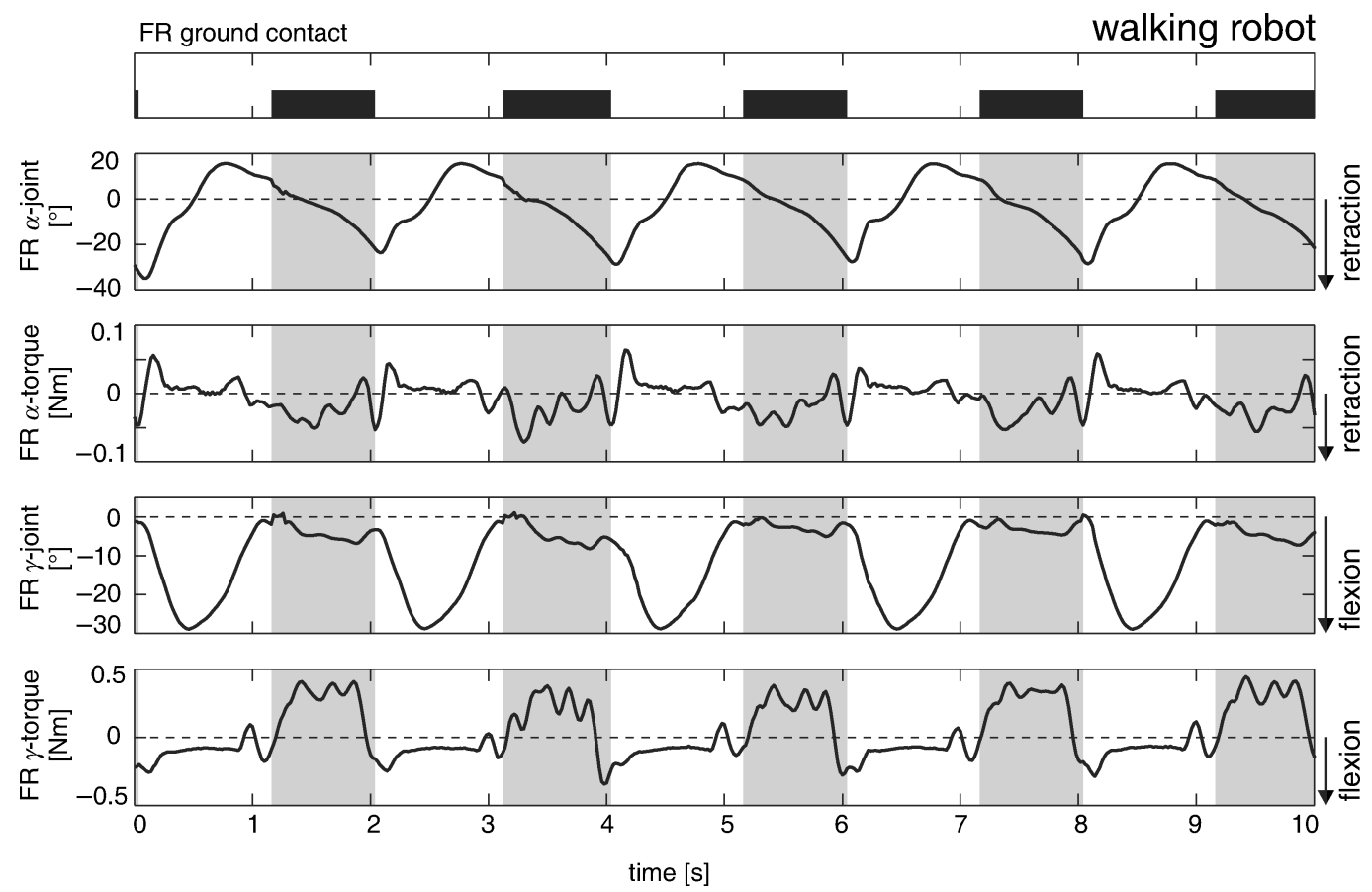

Figure 12. Step pattern of the right front leg of TARRY IIB (top). Below are given the courses of the $\alpha$-joint angle, the torque of the $\alpha$-servo-drive, the $\gamma$-joint angle and the torque of the $\gamma$-servo-drive (from top to bottom). 


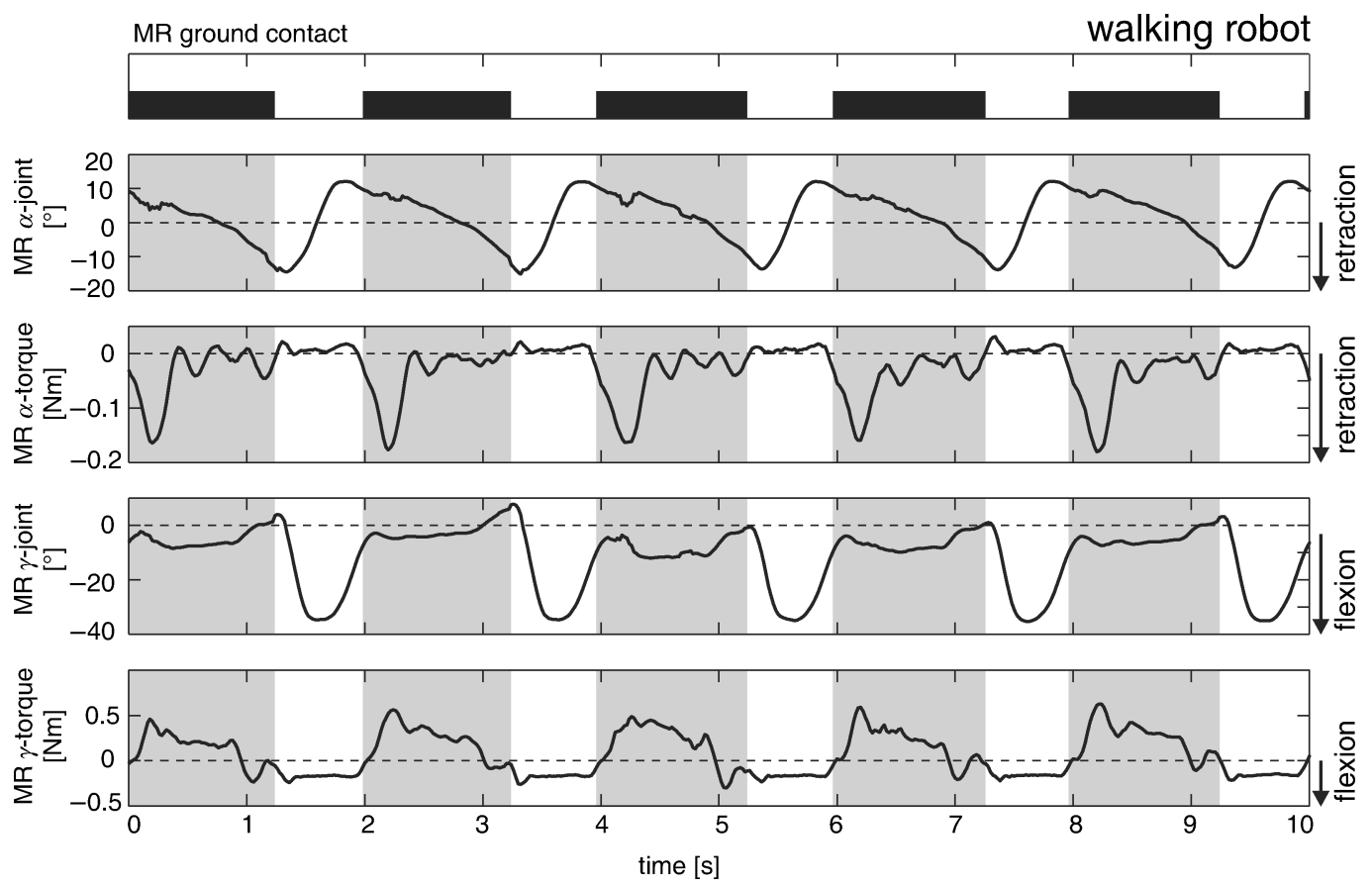

Figure 13. Step pattern of the right middle leg of TARRY IIB (top). Below are given the courses of the $\alpha$-joint angle, the torque of the $\alpha$-servo-drive, the $\gamma$-joint angle and the torque of the $\gamma$-servo-drive (from top to bottom).

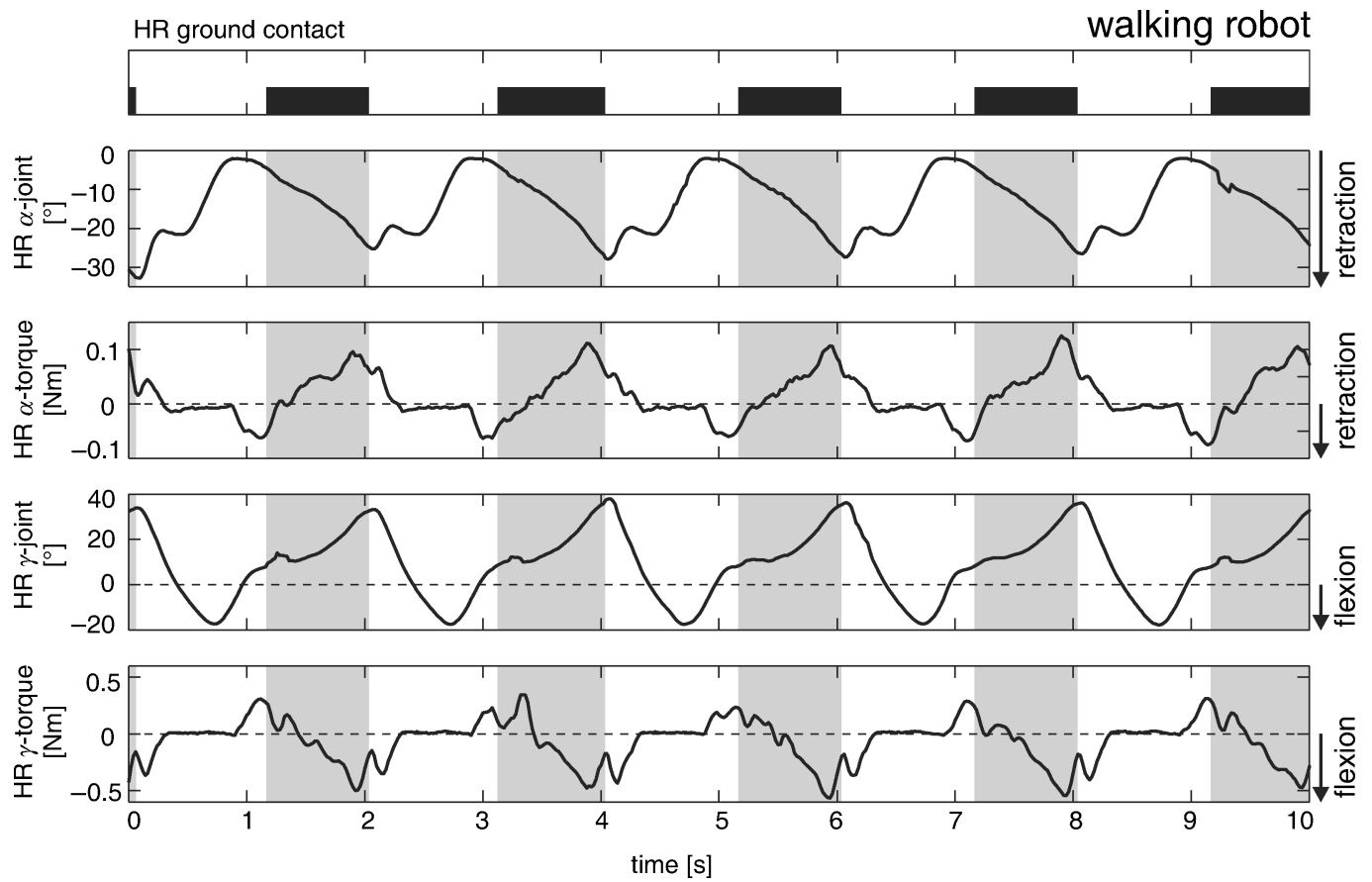

Figure 14. Step pattern of the right hind leg of TARRY IIB (top). Below are given the courses of the $\alpha$-joint angle, the torque of the $\alpha$-servo-drive, the $\gamma$-joint angle and the torque of the $\gamma$-servo-drive (from top to bottom). 
whereas middle legs are flexing during the first part of the stance and then extending towards end of the stance. Hind legs are extending the tibia throughout the whole stance. During all movements appropriate torques are produced, suited to propel the body forward (front and middle legs) and supporting the body weight (hind legs).

\section{Discussion}

Application of LPVF has been proposed to solve the computationally complex task of coordinating up to $18 \mathrm{de}-$ grees of freedom, 12 of which are redundant in a typical hexapod walker. This idea has earlier been tested successfully, however, using a kinematic simulation approach only (Kindermann 2002). Here, we show that this concept still appears to be sensible when dynamical properties are taken into account. Using this concept, the force distribution problem can be solved without a central controller. Instead, each joint controller acts independently and the coupling between these controllers is given by physics, i.e. the geometrical properties of the body and the interaction loop through the environment. Therefore, if the morphology is changed, for example by shortening any leg segment, no reprogramming was necessary. Correspondingly, adaptation to differently shaped substrate is straightforward. Furthermore, a basic form of curve walking is possible simply by readjusting the gain factors of the positive feedback loops differently for legs on the left and on the right side of the body. Tighter curves could be negotiated if front legs would be moved to the side, as has been observed in stick insects, for example by Dürr (2005) and Rosano and Webb (2007).

As an important further result, the simulation shows that the concept of positive velocity feedback is sensible even for quite irregular leg movements as they are also observed in animal walking. Comparison of the behaviour of the simulated system and of stick insect data shows considerable agreement, but also some differences. Differences found for $\gamma$-torques might be explained by the fact that in the simulation the center of body mass is situated between the middle legs and in the robot TARRY IIB between middle and hind legs. In contrast, in stick insects the center of mass is situated between the hind legs. Therefore, hind legs have to carry most of the body weight requiring some flexion torque in the femur-tibia joint $(\gamma)$.

Finally, we would like to point out that the idea to apply positive velocity feedback to control walking is traced back to the observation of Bässler $(1976,1986)$ who described that a resistance reflex, hence negative feedback, found in the femur-tibia joint of an inactive stick insect is eventually changed to an assistance reflex, hence a positive feedback, when the animal is stimulated by a brush, for example to perform active leg movements. Such a reversal was not found in the $\beta$-joint, but has been found in the $\alpha$-joints, too (Schmitz et al. 1995). As this is exactly the result required for application in walking $-\beta$-joints take part in negative feedback height control - the concept of positive velocity feedback was first applied to a legged system using a simple kinematic simulation (Cruse et al. 1995). The final crucial experimental verification of this concept on a physical robot has now been shown in this study.

\section{Acknowledgements}

This work has been supported by the Deutsche Forschungsgemeinschaft (DFG grants Cr58/10-1 and Cr58/11-1) to H.C. and J.S. and a BMBF-BIONA grant (ELAN-project) to A.S.

\section{References}

Bartling C, Schmitz J. 2000. Reactions to disturbances of a walking leg during stance. J Exp Biol. 203:1211-1223.

Bässler U. 1976. Reversal of a reflex to a single motoneuron in the stick insect carausius morosus. Biol Cybern, 24:47-49.

Bässler U. 1986. Afferent control of walking movements in the stick insect cuniculina impigra. J Comp Physiol [A] 158:351362.

Bässler U. 1988. Functional principles of pattern generation for walking movements of stick insect forelegs: The role of the femoral chordotonal organ afferences. J Exp Biol. 136:125147.

Bässler U, Büschges A. 1990. Interneurones participating in the "active reaction" in stick insects. Biol Cybern, 62:529538.

Bässler U, Büschges A. 1998. Pattern generation for stick insect walking movements - multisensory control of a locomotor program. Brain Research Reviews, 27(1):65-88.

Burrows M, Pflüger HJ. 1988. Positive feedback loops from proprioceptors involved in leg movements of the locust. J Comp Physiol A 163:425-440.

Cruse H. 1976. On the function of the legs in the free walking stick insect carausius morosus. J Comp Physiol [A] 112:235-262.

Cruse H. 1990. What mechanisms coordinate leg movement in walking arthropods? Trends in Neurosciences, 13:15-21.

Cruse H, Bartling C. 1995. Movement of joint angles in the legs of a walking insect, carausius morosus. J. Insect Physiol. 41:761771.

Cruse H, Bartling C, Kindermann T. 1995. High-pass filtered positive feedback for decentralized control of cooperation. In Moran F., Moreno A, Merelo JJ, Chacon P. editors. Advances in artificial life, New York: Springer, pp. 668-678.

DiCaprio RA, Clarac F. 1981. Reversal of a walking leg reflex elicited by a muscle receptor. J Exp Biol. 90:197-203.

Dürr V. 2005. Context-dependent changes in strength and efficacy of leg coordination mechanisms. J Exp Biol. 208(12):22532267.

Dürr V, Schmitz J, Cruse H. 2004. Behaviour-based modelling of hexapod locomotion: Linking biology and technical application. Arthropod Struct Devel 33(3):237-250.

Kindermann T. 2002. Behavior and adaptability of a six-legged walking system with highly distributed control. Adapt Behav. 9(1): 16-41,

Klein J. 2003. Breve: a 3D environment for the simulation of decentralized systems and artificial life. In ICAL 2003: 
Proceedings of the 8th International Conference on Artificial life, MIT Press: pages 329-334, Cambridge, MA, USA.

Rosano H, Webb B. 2007. A dynamic model of thoracic differentiation for the control of turning in the stick insect. Biol Cybern, 97(3):229-246.

Schilling M, Cruse H, Arena P. 2007. Hexapod walking: an expansion to capital Walknet dealing with leg amputations and force oscillations. Biol Cybern 96(3):323-340.

Schmitz J, Bartling C, Brunn DE, Cruse H, Dean J, Kindermann T, Schumm M, Wagner H. 1995. Adaptive properties of "hardwired" neuronal systems. In Verh. Dtsch. Zool. Ges. 88.2, pages $165-179$.
Schmitz J, Haßfeld G. 1989. The treading-on-tarsus reflex in stick insects: Phase-dependence and modifications of the motor output during walking. J Exp Biol. 143:373-388.

Schneider A, Cruse H, Schmitz J. 2006. Decentralized control of elastic limbs in closed kinematic chains. The Int J Robotics Res. 25(9):913-930.

Schumm M, Cruse H. 2006. Control of swing movement: influences of differently shaped substrate. J Comp Physiol [A], 192:1147-1164.

Vedel J. 1980. The antennal motor system of the rock lobster: Competitive occurence of resistance and assistance reflex patterns originating from the same proprioceptor. J Exp Biol. 87:1-22. 

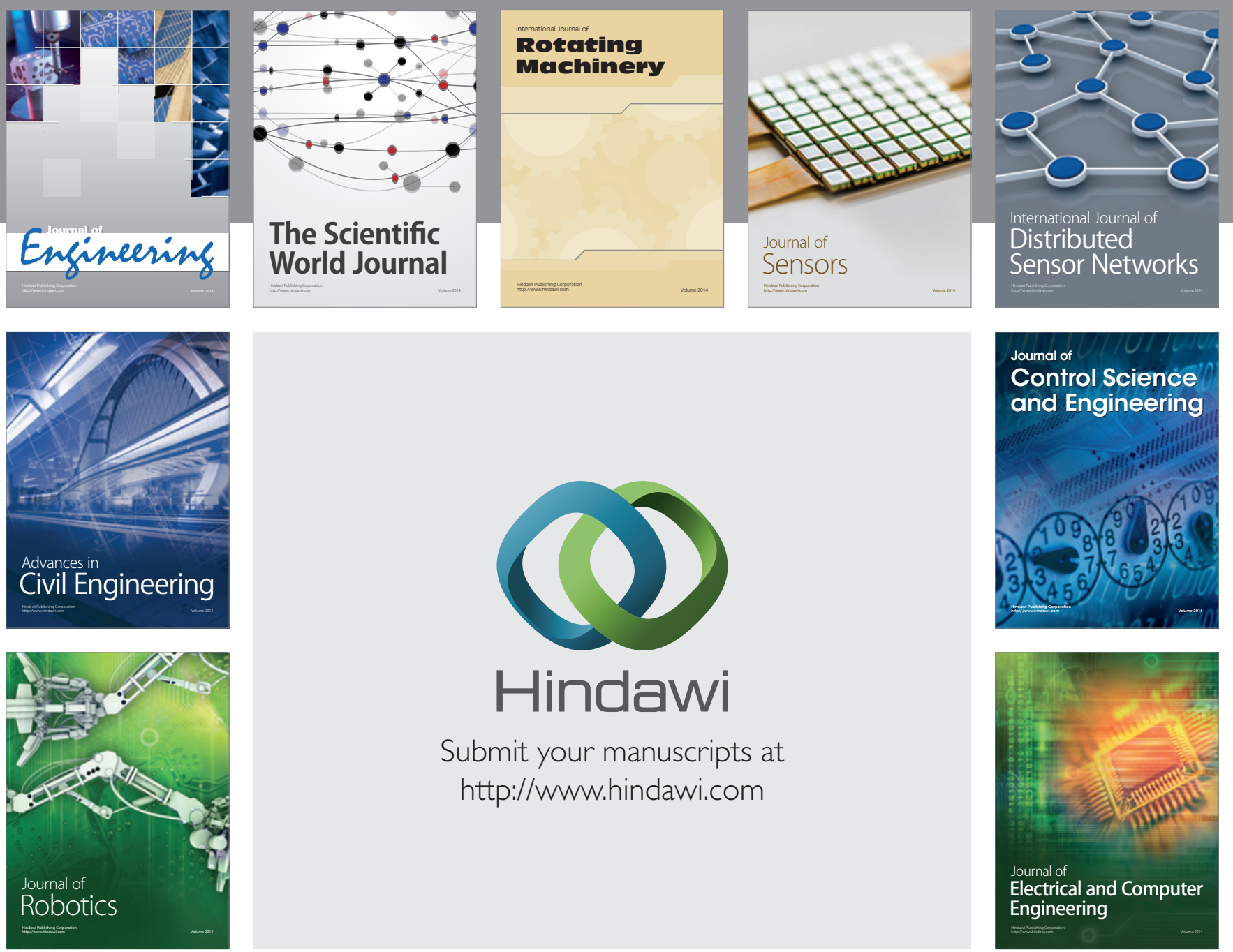

Submit your manuscripts at

http://www.hindawi.com
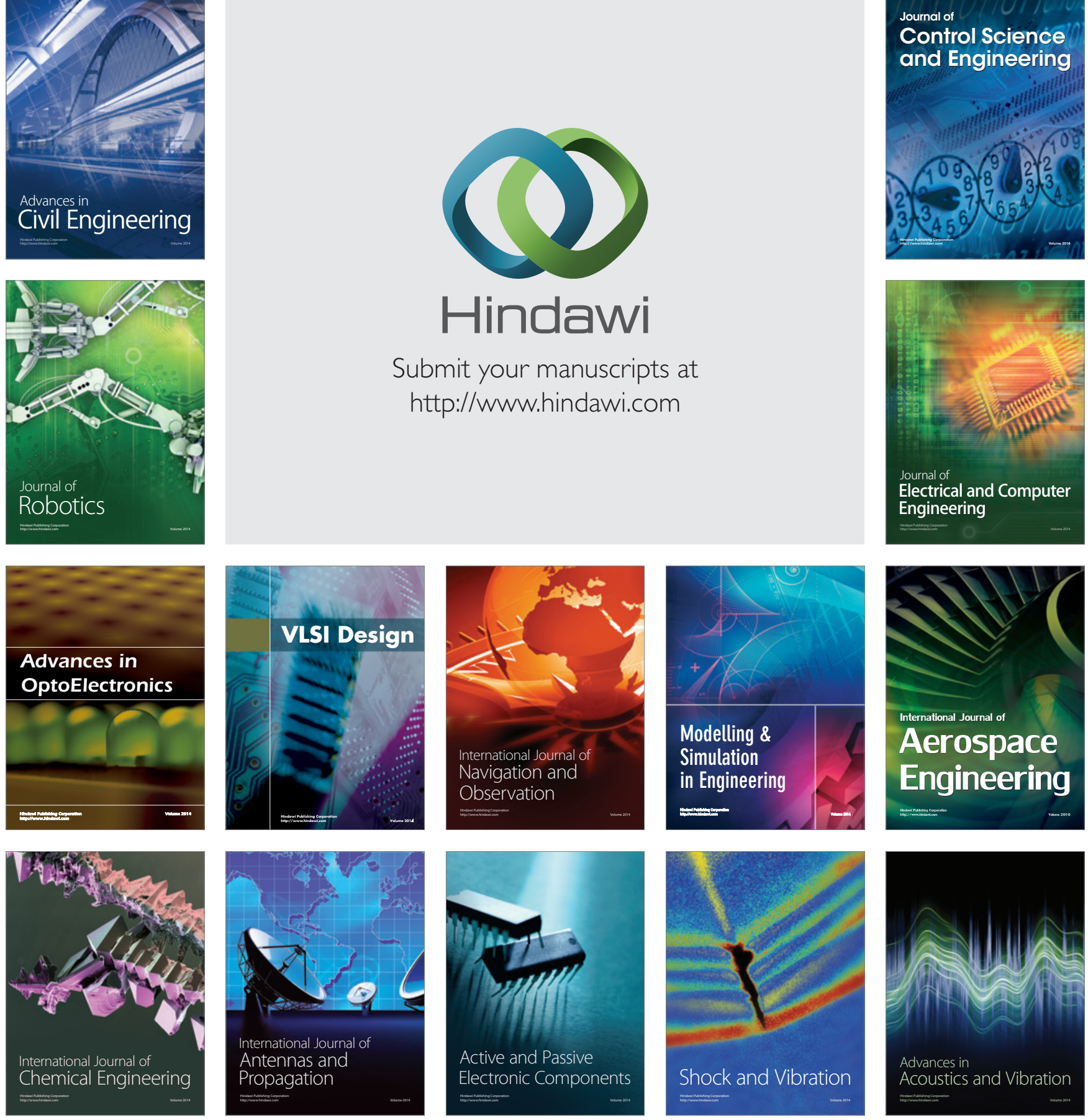